\title{
A Robust Top-Down Approach for Rotation Estimation and Vanishing Points Extraction by Catadioptric Vision in Urban Environment
}

\author{
Jean-Charles Bazin and Inso Kweon \\ RCV Lab, KAIST \\ 373-1 Guseong-dong Yuseong-gu \\ 305-701 Daejeon, Korea \\ jcbazinercv.kaist.ac.kr \\ iskweon@kaist.ac.kr
}

\author{
Cédric Demonceaux and Pascal Vasseur \\ MIS, UPJV \\ 33 Rue Saint Leu \\ 80039 Amiens, France \\ cedric.demonceauxeu-picardie.fr \\ pascal.vasseur@u-picardie.fr
}

\begin{abstract}
A key requirement for Unmanned Aerial Vehicles (UAV) applications is the attitude stabilization of the aircraft, which requires the knowledge of its orientation. It is now well established that traditional navigation equipments, like GPS or INS, suffer from several disadvantages. That is why some works have suggested a vision-based approach of the problem. Especially, catadioptric vision is more and more used since it permits to gather much more information from the environment, compared to traditional perspective cameras, and therefore the robustness of the UAV attitude estimation is improved. Rotation estimation from conventional and catadioptric images has been extensively studied. Whereas interesting results can be obtained, the existing methods have non-negligible limitations such as difficult features matching (e.g. repeated texture, blurring or illumination changing) or a high computational cost (e.g. vanishing point extraction or analyze in frequency domain). In order to overcome these limitations, this paper presents a top-down approach for estimating the rotation and extracting the vanishing points in catadioptric images. This new framework is accurate and can run in real-time. To obtain the ground truth data, we also calibrate our catadioptric camera with a gyroscope. Finally, experimental results on a real video sequence are presented and compared to the ground truth data obtained by the gyroscope.
\end{abstract}

\section{INTRODUCTION}

Automatic stabilization of an Unmanned Aerial Vehicle (UAV) requires the knowledge of the vehicle orientation. It is now well established that traditional navigation equipments, like GPS or INS, suffer from several disadvantages. For example, GPS is sensitive to signal dropout and to hostile jamming. The drawback of INS is that its position error compounds over time and may cause large localization errors. In order to overcome these disadvantages, many researchers suggested a vision-based approach of the navigation problem, which helps estimating localization and/or orientation of a UAV when GPS or inertial guidance is not available [1], [2], [3]. Most of these works use conventional cameras that have a relative small field of view, which drastically limits the information we could get from the environment. At the contrary, catadioptric vision systems usually have a field of view greater than 180 degrees. That is why, they equip more and more robots for ground [4], [5] and aerial [6], [7], [8], [9] applications.

Rotation estimation from conventional and catadioptric images has been extensively studied and the proposed methods can be divided into three main categories. The first one is based on feature correspondence (e.g. Harris corner, SIFT, lines) and epipolar geometry ([10], [11] for conventional cameras, [12], [13], [14] for catadioptric vision). Whereas impressive results can be obtained, this common approach still suffers from several important limitations and difficulties: matching feature points is not an easy task (repeated texture, blurring, illumination changing, high distortions due to the mirror, etc...), different apparent motion in dynamic scenes, the essential matrix is unstable in planar environment and the homography is not defined for non-planar scenes, distant features are usually ineffective, and so on. Moreover these works are indirect in the sense they estimate the complete ego-motion (rotation and translation), although UAV can be stabilized using only rotation information. The second category performs without feature correspondences and refers to some different approaches. For conventional cameras, [15] presents a probabilistic method that samples from the distribution over all correspondence sets using a Markov chain Monte Carlo algorithm. However, the technique assumes that the number of features is known and does not treat outliers and occlusion. For catadioptric vision, [16] converts the image in the frequency domain by spherical Fourrier transform. Then the rotation estimation is refined from the conservation of harmonic coefficients in the rotational shift theorem. Since the whole image is analyzed, the method is computationally expensive. Moreover, it is sensitive to translation and dynamic environment.The third category relies on vanishing points and infinite homography ([17], [18] for conventional cameras, [19], [20] for catadioptric vision). In a control point of view, [21] has shown that points at infinity, and thus infinite homography, yields rotation decoupling properties that respect the UAV dynamics and results in a better behaved control solution. However extracting vanishing points is usually time consuming, which does not permit real-time applications.

In this paper, we aim to estimate the complete rotation in real time. We have privileged catadioptric vision because it provides two important properties for our approach. First, thanks to the wide field of view, it can acquire many parallel lines that lie in different directions. And second, the vanishing points usually lie in the image. Therefore it is possible to detect the vanishing points more accurately. Existing methods usually detect the vanishing points and then 
compute the rotation (bottom-up approach). At the contrary, we introduce a top-down approach that, given a rotation estimation, computes the consistency of the associated vanishing points. As will be explained in details, this new framework provides several advantages: it runs in real-time, ensures orthogonality of vanishing points, can use a priori rotation information (e.g. from the current GPS/INS data or the visual information of the previous frame) and is very robust. In order to show the accuracy of the proposed method, we also calibrate our camera with a gyroscope. Finally, experimental results on a real video sequence are presented and compared to ground truth data.

\section{CATADIOPTRIC VISION AND IMAGE FORMATION}

A catadioptric vision system is composed of a mirror with a specific shape and a conventional camera. Such systems have a field of view of 360 degrees in horizontal direction and greater than 180 degrees in vertical direction. Geyer and Daniilidis have demonstrated the equivalence for the central catadioptric sensors (single viewpoint constraint) with a two-step projection via a unitary sphere centered on the focus of the mirror [22]. Whereas it does not seem obvious at the first sight, the sphere equivalence is interesting for several reasons. First, it greatly simplifies the formalism to take catadioptric distortions into account. Indeed catadioptric images are highly distorted because of the mirror projection, which complicates the image analysis. Working in the sphere permits to manipulate those distortions much more easily. A second reason is that it allows working in a general framework, i.e. the sphere, independently on the fact that a hyperbolic or a parabolic mirror is used. Finally, the sphere space provides some very interesting projection properties. For example, it has been proved that a line is simply projected as a great circle in the sphere [22], which is used by several line detection algorithms. A second important projection property is that the great circles associated to a pencil of 3D parallel lines intersect in two antipodal points in the sphere.

\section{ROTATION ESTIMATION}

The common approach for estimating the rotation from vanishing points consists in three consecutive main steps: first, the lines are extracted (denoted as step 1); second, the parallel lines are grouped together and the corresponding vanishing points are computed (denoted as step 2); and thirdly, the rotation is estimated from the infinite homography (denoted as step 3). For step 1, we have used our algorithm [19]. The main idea is the following: after detecting edges in the image and building chains of connected edge pixels, we project these chains on the sphere and check whether they verify the great circle constraint, that is to say whether they correspond to projection of world lines. For this, we apply a split and merge algorithm based on the distance between chain points and the plane defining a great circle. The algorithm returns a list of great circle normals corresponding to the detected lines. It has the advantages of running very fast with accurate results. Moreover it can handle large occlusion and perform for general central catadioptric images.

In the next subsections, we first make a review of existing systems that estimate rotation from lines and then we present our method.

\section{A. Previous work based on vanishing points}

Given a set of lines, many methods have been proposed for grouping parallel lines and computing their vanishing points (step 2). Most of them perform on the Gaussian sphere where parallel lines are projected in great circles that intersect in two antipodal points (fig 1). For all line pairs, [23] computes a cross-product which corresponds to a direction parameterized by the azimuth and elevation angles $(\theta, \phi)$ in sphere space. The dominant vanishing point is obtained by sampling the $(\theta, \phi)$ parameter space and selecting $\left(\theta_{\max }, \phi_{\max }\right)$ that contains the highest number of entries. This basic approach suffers from several important limitations. First, it is computationally expensive since it has a complexity of $N\left(\frac{N-1}{2}\right)$ where $N$ is the number of lines. Second, it is sensitive to the sampling of the parameter space. Moreover, it does not directly impose orthogonality constraint on vanishing points since they are independently detected. Indeed, to find an additional vanishing point, the $\left(\theta_{\max }, \phi_{\max }\right)$ corresponding to the second highest number of entries is considered or the algorithm is simply run again without the line segments belonging to the previously detected vanishing point, the latter approach providing much better results. Finally, $a$ priori information about vanishing directions or rotation can not be used. For every pair of lines, [19] computes the associated direction $\vec{d}$ by cross-product and then for each line, measures the distance to $\vec{d}$. If the measurement is smaller than a certain threshold, then the lines are considered parallel and their associated vanishing points is $\vec{d}$. This approach, avoiding sphere sampling difficulty but having a complexity of $N(N-2)\left(\frac{N-1}{2}\right)$, do not impose orthogonality constraint and cannot use a priori information. [24] presents a hierarchical Hough transform on the sphere. It is faster than the classical Hough transform (HT) and has a worst complexity of $O\left(\mathrm{~m}^{2}\right)$ where $m$ is the parameter sampling rate. However, it does not directly impose orthogonality constraint and cannot use a priori information. In [20], the authors first perform the Hough transform directly on edge chains (i.e. not on intersection of line pairs) and thus obtain a linear complexity. The results are pretty inaccurate but the authors argue that they can be used as an initialization for an Expectation-Maximization (EM) algorithm to refine the estimation of vanishing points and the parallel lines grouping. While interesting results have been obtained, this approach assumes that the initialization provided by HT is accurately enough for EM convergence. Moreover Horn's method is directly applied on extracted vanishing points whereas they might not be orthogonal. Finally, a magic number is used as a key parameter in the modified HT to determine the crucial number of mixtures for the EM algorithm. 


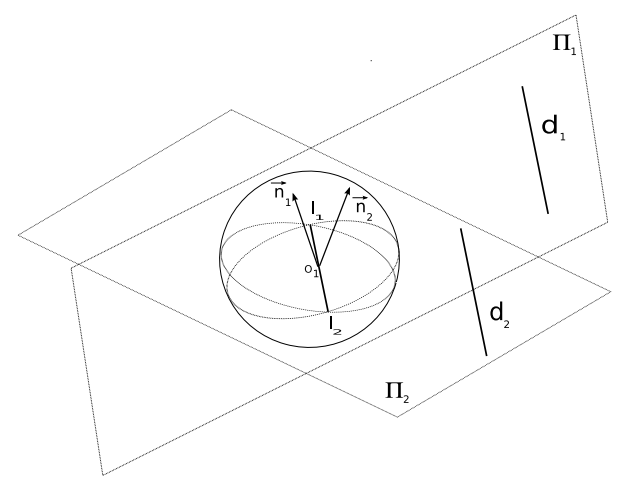

Fig. 1. The direction of two parallel lines corresponds to the direction of their associated vanishing points in the sphere

Therefore it appears that existing methods are too computationally expensive, cannot use a priori information, do not impose orthogonality constraints and/or are sensitive to parameters sampling. Moreover they do not guarantee a constant angle between vanishing points in a video sequence. For example, in Manhattan-like environment, the orthogonality of vanishing points must be imposed along the whole sequence. Regarding step 3, a set of vanishing points correspondences permits to easily compute the best rotation. Traditionally, this system is non-linear with respect to the three rotation angles but [25] has published a closed-form solution based on unit quaternions.

\section{B. Proposed method for rotation estimation}

As reviewed, the existing methods consist in independently extracting the vanishing points and then compute the rotation (bottom-up approach). In order to overcome the limitations associated to this approach (computationally expensive, nonorthogonality, no use of a priori rotation information, etc...), we propose to invert the problem: we first make hypothesis on the rotation and then we evaluate the consistency of the vanishing points extraction (top-down approach). This method can be formalized as the maximization of the following function:

$$
\arg \max _{\alpha, \beta, \gamma} \sum_{i=1}^{3} \sum_{j=1}^{N} \delta\left(R_{\alpha, \beta, \gamma} \overrightarrow{e_{i}}, \overrightarrow{n_{j}}\right)
$$

with

$$
\delta(\vec{u}, \vec{v})= \begin{cases}1 & \text { if }\left|\arccos (\vec{u} \cdot \vec{v})-\frac{\pi}{2}\right| \leq \tau \\ 0 & \text { otherwise }\end{cases}
$$

where $\overrightarrow{e_{i}}$ represents the $i^{t h}$ axis of the Cartesian coordinate system (i.e. $\left.\overrightarrow{e_{1}}=(1,0,0)\right), \overrightarrow{n_{j}}$ the normal associated to the $j^{\text {th }}$ detected line in sphere space (obtained by [19]), $N$ the number of detected lines, $\alpha, \beta, \gamma$ correspond to roll, pitch and yaw angles and $R_{\alpha, \beta, \gamma}$ is the associated rotation matrix.

The proposed function can be read as follows: an original world coordinate system $\left(\overrightarrow{e_{1}}, \overrightarrow{e_{2}}, \overrightarrow{e_{3}}\right)$ is transformed by a certain rotation $R_{\alpha, \beta, \gamma}$. If this rotation corresponds to the rotation of the camera with respect to the original world frame, then the newly obtained coordinate system must be aligned with the dominant directions of the image (i.e. the vanishing points). In order to check this alignment, we simply count the number of lines that are in a direction similar to the axis of this new frame, where $\tau$ defines the angle similarity threshold. This is done by the Dirac $\delta$ function which computes the angle between each $\overrightarrow{n_{j}}$ with the $R_{\alpha, \beta, \gamma} \overrightarrow{e_{i}}$ for each direction, i.e. for $i=1,2,3$. For fast execution purpose, one might prefer the equivalent Dirac condition $|\vec{u} \cdot \vec{v}| \leq \tau$, where $\tau$ defines the dot product similarity threshold. A discussion about the original world coordinate system $\left(\overrightarrow{e_{1}}, \overrightarrow{e_{2}}, \overrightarrow{e_{3}}\right)$ and the number of observable directions is performed in section VI-B. When maximization of equation (1) is completed, we obtain:

- the best absolute rotation matrix: $R_{\alpha_{\max }, \beta_{\max }, \gamma_{\max }}$

- the lines that are in the same direction as the rotated axis. These three sets of lines correspond to parallel lines

- the three vanishing points $R_{\alpha_{\max }, \beta_{\max }, \gamma_{\max }} \vec{e}_{i}$. Note that they are mutually orthogonal by construction, since the basis $\left(\overrightarrow{e_{1}}, \overrightarrow{e_{2}}, \overrightarrow{e_{3}}\right)$ is orthogonal.

In an initial approach, we had maximized this function by a simple exhaustive search on $\alpha, \beta$ and $\gamma$ angles. $A$ priori rotation information (e.g. results of the previous frame) provides initial values $\alpha_{e s t}, \beta_{e s t}$ and $\gamma_{e s t}$. We defined the associated search space of each estimated angle angle $e_{\text {est }}$ as $\left\{\right.$ angle $\left._{\text {est }}-D+k T\right\}$ with integer $k \in[0,2 D / T]$, i.e. the interval centered at angle est with an offset of $\pm D$ and a sampling rate of $T$. Therefore the total number of dot products to compute is $3 N\left(\frac{2 D}{T}\right)^{3}$, where $N$ is the number of detected lines. As $T$ is fixed, we refer this method as the fixed sampling algorithm (FS). Experiments have shown that FS can run very fast with some parameters (such as $T=2^{\circ}$ and $D=5^{\circ}$ ) but is too slow for parameters defining a large interval with a very fine sampling (e.g. $T=1^{\circ}$ and $D=10^{\circ}$ ). That is why, in our final implementation, we have preferred a multi-scale sampling approach: the function (1) is maximized by successively refining the discretization of the rotation angles until a desired precision is reached (cf algorithm 1). This method permits to avoid an exhaustive search on very large and fine intervals while maintaining a given accuracy. At any accuracy level $J$, the search space of an angle is split in $K$ intervals, thus the complexity is $3 N(K+1)^{3}$. The maximum accuracy level to reach an accuracy $T_{\text {goal }}$ is $J_{\text {max }}=\operatorname{ceil}\left(\ln \left(\frac{T_{\text {goal }}}{D}\right) / \ln \left(\frac{2}{K}\right)\right)=$ ceil $\left(\log _{2 / K}\left(\frac{T_{\text {goal }}}{D}\right)\right)$ where ceil is the round toward infinity operator. Therefore the total complexity is $\sum_{J=1}^{J_{\max }} 3 N(K+1)^{3}$.

\section{CAMERA-GYROSCOPE CALIBRATION}

In order to analyze the accuracy of the proposed method, we propose to compare the computed rotation angles with ground truth data obtained by a gyroscope rigidly attached to the camera. The gyroscope returns the orientation between its own frame and an Earth coordinate system composed of magnetic North, West and opposite gravity directions. At 


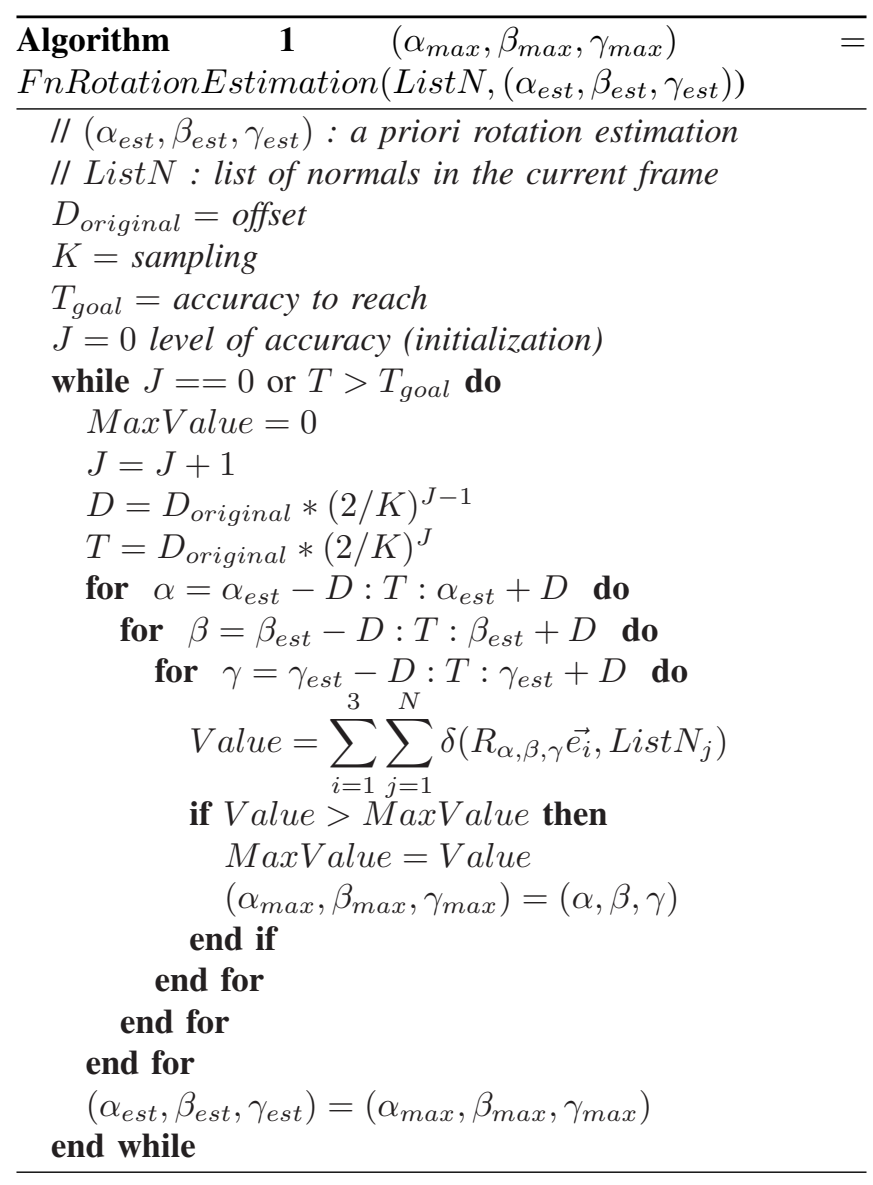

the contrary, our method computes the orientation between the camera frame and the coordinate system composed of the detected vanishing directions. That is why the results obtained from these two sensors cannot be directly compared. In order to calibrate an Inertial Measurement Unit (IMU) with a camera, [26] estimates the relative rotation by having both sensors observing the vertical direction at different poses (fig 2). Vertical is obtained from the camera by using a chessboard target and from the IMU by magnetic field information. However our gyroscope only provides absolute angles and thus this method cannot be directly applied. Nevertheless, the knowledge of the orientation angles $(\alpha, \beta$ and $\gamma$ ) permits to recompute the vertical direction $n=\left(n_{x}, n_{y}, n_{z}\right)$ in the gyroscope frame by the following relation:

$$
\left(\begin{array}{l}
n_{x} \\
n_{y} \\
n_{z}
\end{array}\right)=R_{\alpha, \beta, \gamma}^{T}\left(\begin{array}{l}
0 \\
0 \\
1
\end{array}\right)
$$

The vertical direction in the image could be computed from a specific target (e.g. chessboard pattern), but it is not very convenient. We preferred to run the algorithm presented in section III-B, and select the vertical among the three detected directions. In theory, such a procedure would also be possible for conventional cameras but in practice, it is complicated because of the limited number of observable lines, the sensitivity to measurement noise and the fact that

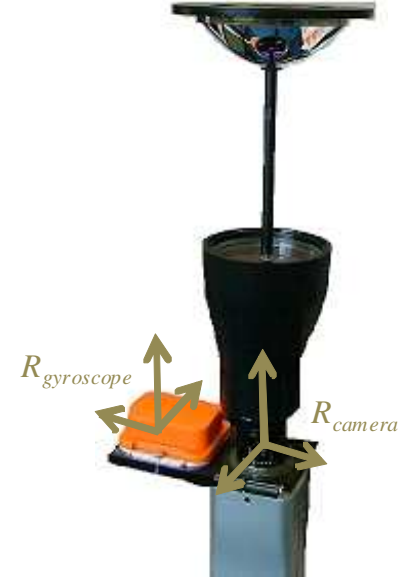

Fig. 2. The coordinate systems of the camera and the gyroscope

the vanishing points do not lie in the image. Given the vertical direction in the gyroscope and camera frames, we simply have to find the unit quaternion $\dot{q}$ that maximizes:

$$
\sum_{i=1}^{n}\left(\dot{q} n_{i} \dot{q}^{*}\right) \cdot v_{i}
$$

where $v_{i}$ and $n_{i}$ respectively represent the vertical in the camera and the gyroscope frames obtained by (eq 3). This system is easily solved by [25] and permits to obtain the calibration rotation matrix $R_{\text {calib }}$. Therefore any vectors in the camera frame can now be projected to the gyroscope frame. By construction, the projection of the vertical direction $V$ obtained by the camera will be aligned with the gravity $G$ obtained by the gyroscope but the two horizontal directions $\left(H_{1}\right.$ and $\left.H_{2}\right)$ obtained by the camera will not be aligned with the North and West directions $(N$ and $W$ ). If we project $V, H_{1}$ and $H_{2}$ in the Earth frame by successively applying $R_{\text {calib }}$ and $R_{\text {gyroscope }}$, one may compute the relative rotation $R_{r e l}$ around the vertical that maps the projected $V, H_{1}$ and $H_{2}$ to $G, N$ and $W$. Finally, we are now able to compare $R_{\text {gyroscope }}$ and $R_{\text {camera }}$ by verifying the following relation:

$$
R_{\text {gyroscope }}=R_{\text {rel }}^{T} R_{\text {cam }} R_{\text {calib }}^{T}
$$

\section{EXPERIMENTAL RESULTS}

In this section, we present some experimental results concerning camera-gyroscope calibration and the rotation estimation using the proposed approach.

\section{A. Camera-gyroscope calibration}

As explained in section 4, the camera-gyroscope calibration method is based on the vertical directions observed from the gyroscope and the camera. Figure 3 depicts these measurements. The quaternion obtained by [25] corresponds to the following relative angles: roll $=-2.82^{\circ}$, pitch $=$ $0.01^{\circ}$ and yaw $=-179.1^{\circ}$, i.e. a near $180^{\circ}$ rotation about the camera $\mathrm{z}$-axis, which is consistent to the layout of our system. Comparing the gyroscope vertical with the newly 

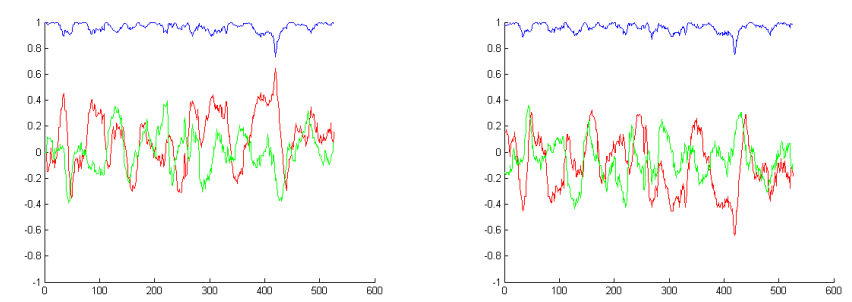

Fig. 3. Observation of the vertical direction from the gyroscope (left) and the camera (right) with sampling parameters $T=1^{\circ}$ and $D=5^{\circ}$. The 3 coordinates $\left(v_{x}, v_{y}, v_{z}\right)$ of the vertical direction are respectively displayed in red, green and blue. One may already notice the relationship between these two sensors: a near $180^{\circ}$ rotation about the camera z-axis

calibrated camera vertical, we have obtained a mean error of $2.0^{\circ}$ with a standard deviation of $1.1^{\circ}$. Finally, the computed relative rotation $R_{\text {rel }}$ between Earth frame and the vanishing directions corresponds to a rotation of $58^{\circ}$ around the vertical. On a Google Earth image of the scene, we have manually extracted an horizontal direction of buildings and the North direction: we have obtained an angle of $62^{\circ}$ which is consistent with the estimated $R_{\text {rel }}$.

\section{B. Rotation estimation}

To analyze our method, we have acquired a video sequence composed of 520 frames at 5 frames per second and the associated gyroscope data. The video was acquired in the parking lot of our laboratory (i.e. a typical urban scene). The total travel path is about 40 meters and the rotation amplitude is about $40^{\circ}$ for roll, $60^{\circ}$ for pitch and $360^{\circ}$ for yaw angles. For each frame of the sequence, we estimate the best rotation by optimizing equation 1 . The results of the previous frame are used to reduce the search space and for the first frame, an initial solution is obtained by the hierarchical Hough transform of [24] (cf section III-a). Figure 4 depicts typical results of vanishing points extraction for this sequence and figure 5 shows the rotation angles estimated by our proposed method and the gyroscope ground truth data. For qualitative comparison, table I contains the error between the gyroscope data and our proposed method with different sets of sampling parameters. These results demonstrate the accuracy of our approach. The vanishing points obtained by our method are mutually orthogonal by construction whereas data analysis for [19] have shown that the average angle is $89.7^{\circ}$ with a standard deviation of $4.7^{\circ}$.

We have also applied the proposed algorithm on three other sequences with no gyroscope data. The output videos can be downloaded on our website http://rcv.kaist.ac.kr/ jcbazin/ and some typical results are displayed on figure 6. The sequences gungdongl and gungdong2 have been acquired in a dense urban environment and the rotation is estimated by tracking 2 vanishing points (cf discussion VI-A). The sequence chungdael has been recorded on a road. Whereas only one vanishing point can be tracked, we can still compute the rotation by assuming the planar motion of a car on the road since the rotation has only one degree of freedom in this case. The algorithm manages
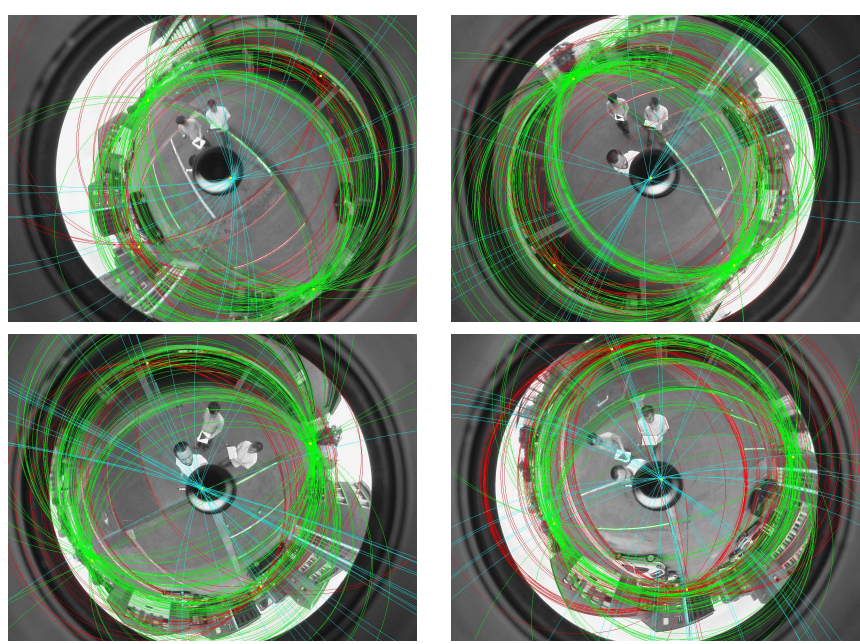

Fig. 4. Experimental results obtained by the proposed method for the extraction of vanishing points and parallel lines. Each conic corresponds to a detected line and all parallel lines have the same color

\begin{tabular}{|c|c|c|c|c|}
\hline algo & parameters & roll & pitch & yaw \\
\hline FS & $T=5, D=10$ & $1.9 / 2.2$ & $2.0 / 1.7$ & $4.3 / 3.1$ \\
\hline MS & $K=4$ & $1.9 / 2.2$ & $2.0 / 1.7$ & $4.3 / 3.1$ \\
\hline MS & $K=10$ & $1.3 / 1.1$ & $1.5 / 1.1$ & $4.0 / 2.9$ \\
\hline FS & $T=2, D=5$ & $1.9 / 2.0$ & $2.2 / 3.2$ & $4.5 / 3.8$ \\
\hline MS & $K=4$ & $2.0 / 3.2$ & $1.7 / 2.0$ & $4.4 / 3.6$ \\
\hline MS & $K=10$ & $1.7 / 1.7$ & $2.0 / 2.8$ & $3.8 / 2.7$ \\
\hline FS & $T=1, D=5$ & $1.7 / 1.7$ & $2.0 / 2.8$ & $3.8 / 2.7$ \\
\hline MS & $K=4$ & $1.3 / 1.2$ & $1.5 / 1.5$ & $4.0 / 2.9$ \\
\hline MS & $K=10$ & $1.7 / 1.7$ & $2.0 / 2.8$ & $3.8 / 2.7$ \\
\hline FS & $T=1, D=10$ & $1.2 / 1.0$ & $1.3 / 1.0$ & $3.9 / 2.8$ \\
\hline MS & $K=4$ & $1.3 / 1.0$ & $1.3 / 1.0$ & $3.7 / 2.7$ \\
\hline MS & $K=10$ & $1.3 / 1.0$ & $1.3 / 1.0$ & $3.8 / 2.7$ \\
\hline [19] & & $4.6 / 2.2$ & $4.3 / 2.1$ & $4.6 / 3.5$ \\
\hline
\end{tabular}

TABLE I

MEAN AND STANDARD DEVIATION ERROR (MEAN/STD) IN DEGREES BETWEEN THE CALIBRATED GYROSCOPE DATA AND THE PROPOSED METHODS OF FIXED SAMPLING (FS) AND MULTI-SCALE SAMPLING (MS) WITH THE SAME SET OF PARAMETERS THAN TABLE II. FOR COMPARISON WITH EXISTING METHODS, WE HAVE ALSO RUN THE ALGORITHM [19].

to track the vanishing points correctly in the 3 sequences. We have also experimented that our approach can handle line outliers (i.e. some lines that are classified to a wrong vanishing point), blurry images (as long as an edge operator can extract some line edge pixels) and dynamic environments (car, pedestrian, etc...) which demonstrates the robustness of our approach.

The execution time of our methods is displayed in table II. The parameters $\left(T=1^{\circ}, D=5^{\circ}\right)$ or $\left(T=1^{\circ}, D=10^{\circ}\right)$ are realistic values for UAV control and $3 \mathrm{D}$ reconstruction, and lead to an average processing time between $57 \mathrm{fps}(K=10)$ and 407 fps $(K=4)$ with $N=100$ lines on non-optimized $\mathrm{C}++$ code. The parameter $\tau$ of equation 1 has been fixed to $2^{\circ}$ and experiments have shown that its value does not play an important role as long as it is physically meaningful such as $\tau \in\left[1^{\circ}, 3^{\circ}\right]$. 

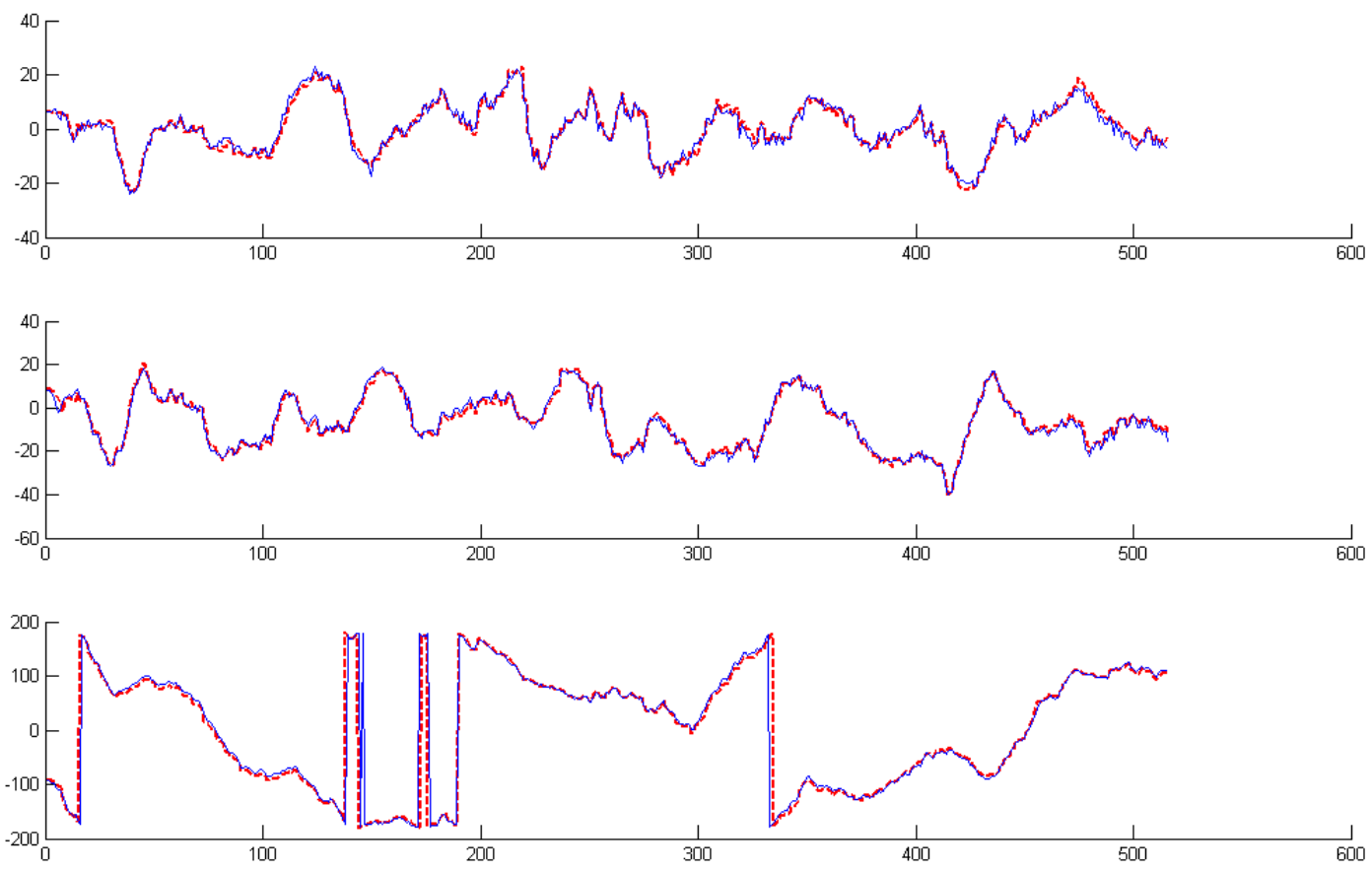

Fig. 5. Comparison of roll (top), pitch (middle) and yaw (bottom) angles between gyroscope (red dashed line) and the proposed method (blue solid line with $\left.T_{\text {goal }}=1^{\circ}, D_{\text {original }}=10^{\circ}, K=10\right)$. The "jumps" of the yaw angle are simply due to the discontinuities $\left(-180^{\circ},+180^{\circ}\right)$.

\begin{tabular}{|c|c|c|c|c|}
\hline algo & parameters & $\mathrm{N}=$ & $\mathrm{N}=$ & $\mathrm{N}=$ \\
& & 50 & 100 & 150 \\
\hline FS & $T=5^{\circ}, D=10^{\circ}$ & 1901 & 1220 & 885 \\
MS & $K=4\left(T_{1}=5, T_{f}=5\right)$ & 1901 & 1221 & 884 \\
MS & $K=10\left(T_{1}=2, T_{f}=2\right)$ & 177 & 114 & 83 \\
\hline FS & $T=2^{\circ}, D=5^{\circ}$ & 1090 & 704 & 512 \\
MS & $K=4\left(T_{1}=2.5, T_{f}=1.25\right)$ & 951 & 608 & 443 \\
MS & $K=10\left(T_{1}=1, T_{f}=1\right)$ & 177 & 114 & 83 \\
\hline FS & $T=1^{\circ}, D=5^{\circ}$ & 177 & 114 & 83 \\
MS & $K=4\left(T_{1}=2.5, T_{f}=0.625\right)$ & 628 & 407 & 296 \\
MS & $K=10\left(T_{1}=1, T_{f}=1\right)$ & 177 & 114 & 83 \\
\hline FS & $T=1^{\circ}, D=10^{\circ}$ & 26 & 17 & 12 \\
MS & $K=4\left(T_{1}=5, T_{f}=0.625\right)$ & 472 & 304 & 221 \\
MS & $K=10\left(T_{1}=2, T_{f}=0.4\right)$ & 89 & 57 & 42 \\
\hline
\end{tabular}

\section{TABLE II}

AVERAGE EXECUTION TIME IN FRAMES PER SECOND (FPS) ON NON-OPTIMIZED C++ CODE WITH RESPECT TO SEARCH SPACE SAMPLING AND NUMBER OF LINES B Y FIXED SAMPLING (FS) AND MULTI-SCALE SAMPLING (MS) ALGORITHMS (EXECUTION TIME FOR LINE EXTRACTION BY [19] NOT INCLUDED).

\section{DISCUSSION}

\section{A. Observability of dominant directions}

The optimization function (1) counts the number of lines that are in the same direction as the 3 rotated axis. One may argue that even in urban environment, there exist some situations where only 2 dominant directions might be observed, instead of 3 . For example, let us imagine that only the green and blue horizontal directions of figure 4 are observed but not the red vertical direction (one may actually select any two directions out of the three). It means there are no lines in the vertical direction and thus only the lines in the two orthogonal horizontal directions will be counted. To verify the robustness of our approach in such a situation, we have slightly modified our optimization function such that it counts the number of lines with respect to two axis only, i.e. $i \in 1,2$ in equation 1 . The complexity decreases to $2 N\left(\frac{2 D}{T}\right)^{3}$ and we have compared the two horizontal directions estimated by using 2 or 3 axis. We have obtained a mean difference of $0.2^{\circ}$ with a standard deviation of $0.2^{\circ}$ for the $1^{\text {st }}$ horizontal direction and a mean difference of $0.3^{\circ}$ with a standard deviation of $0.2^{\circ}$ for the $2^{\text {nd }}$ horizontal direction. It shows that our method can work in the case where only 2 directions are observed.

\section{B. Original reference frame}

The optimization function (1) aims to compute the rotation matrix that best aligns an original world coordinate system $\left(\overrightarrow{e_{1}}, \overrightarrow{e_{2}}, \overrightarrow{e_{3}}\right)$ with the dominant directions of the image. It is usually considered that $\overrightarrow{e_{1}}$ and $\overrightarrow{e_{2}}$ define the ground plane and $\overrightarrow{e_{3}}$ the vertical. Therefore one might expect that the vertical direction of the image will be aligned with $\overrightarrow{e_{3}}$. Whereas it is the case in our experiments, that might not always be true in practice. Indeed, as depicted by figure 7, there exist 3 possible configurations that can align the original world coordinate system with the 3 main directions of the image (6 configurations in case of 2 main directions). Therefore, we have tested our algorithm with the 3 possible direct basis: $\left(\overrightarrow{e_{1}}, \overrightarrow{e_{2}}, \overrightarrow{e_{3}}\right),\left(\overrightarrow{e_{2}}, \overrightarrow{e_{3}}, \overrightarrow{e_{1}}\right)$ and $\left(\overrightarrow{e_{3}}, \overrightarrow{e_{1}}, \overrightarrow{e_{2}}\right)$. As expected, we have obtained different maximizing rotations (since the basis are different), exactly the same number of lines in the directions of the rotated axis and the same vanishing points. 


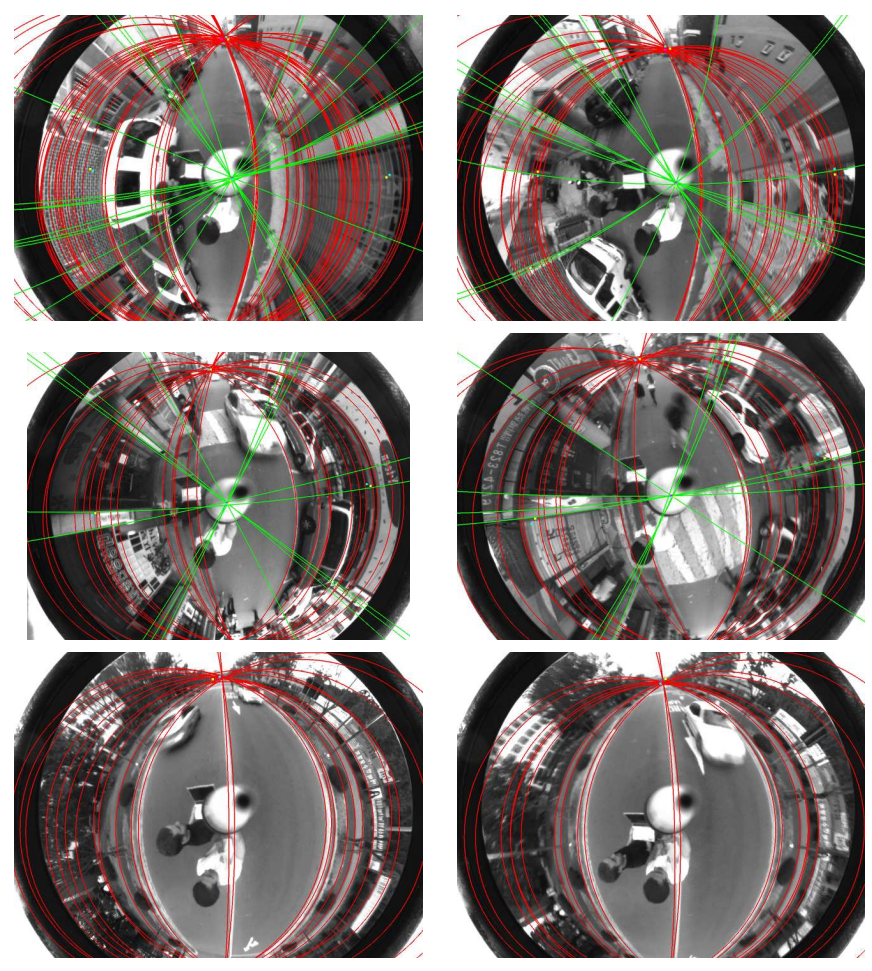

Fig. 6. Vanishing points extraction by the proposed algorithm on the sequences gungdong1 (top-row), gungdong2 (middle-row) and chungdae1 (bottom-row). These sequences contain dynamic environments and blurred images. The conics have been enlarged for a better visualization. More results on http://rcv.kaist.ac.kr/ jobazin/

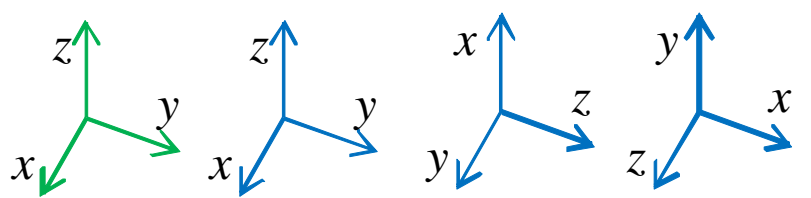

Fig. 7. There exist 3 possible configurations $\left(2^{n d}, 3^{r d}\right.$ and $4^{\text {th }}$ figure) that can align the original world coordinate system with the 3 main directions of the image ( $1^{\text {st }}$ figure).

\section{CONCLUSION}

In this paper, we have presented a top-down framework for rotation estimation and vanishing points extraction in catadioptric images. We have privileged catadioptric vision because it can acquire many parallel lines that are in different directions and the vanishing points usually lie in the image. Therefore the reliability problems of vanishing directions estimation and insufficient support by line segments can be substantially overcome, especially in urban environment, as shown by our experiments. The proposed approach provides several advantages: it runs in real-time, ensures orthogonality of vanishing points, can use a priori rotation information and is robust to line outliers, dynamic environments and blurry images. To validate our method, we have calibrated a gyroscope with our catadioptric camera to obtain ground truth data. Finally, experimental results on real video sequence have demonstrated the accuracy and the robustness of the proposed top-down framework for rotation estimation, parallel lines grouping and vanishing points extraction. In future work, we aim to use our method for fast and robust $3 \mathrm{D}$ reconstruction and also implement an optimized code on a real-time embedded system for UAV control.

\section{ACKNOWLEDGMENTS}

This work has been performed within the STAR project of the Hubert Curien (Egide) partnership between RCV Lab at KAIST-Korea and MIS at UPJV-France. The authors gratefully acknowledge the contribution of Pierre-Yves Laffont from INSA Lyon France for his C++ implementation of our line detection and vanishing points extraction algorithms during his internship at RCV Lab, KAIST, Korea.

\section{REFERENCES}

[1] P.C. Naval, M. Mukunoki, M. Minoh, and K. Ikeda. Estimating camera position and orientation from geographical map and mountain image. In 38th Research Meeting of the Pattern Sensing Group, Society of Instrument and Control Engineers, 1997.

[2] K. Rushant and L. Spacek. An autonomous vehicle navigation system using panoramic machine vision techniques. In Technical report CSM298, 1997.

[3] L.K. Wang, S.C. Hsieh, E.C. Hsueh, F.B. Hsaio, and K.Y. Hunag. Complete pose determination for low altitude unmanned aerial vehicle using stereo vision. In Proceedings of the International Conference on Intelligent Robots and Systems (IROS'05), pages 316-321.

[4] N. Winters, J. Gaspar, G. Lacey, and J. Santos-Victor. Omni-directional vision for robot navigation. In ICCV Workshop on Omnidirectional Vision, Camera Networks and Non-classical Cameras (OMNIVIS'O0).

[5] Peng Chang and Martial Hebert. Omni-directional visual servoing for human-robot interaction. In Proceedings of the International Conference on Intelligent Robots and Systems (IROS'98), volume 3, pages $1801-1807$.

[6] C. Demonceaux, P. Vasseur, and C. Pégard. Robust attitude estimation with catadioptric vision. In Proceedings of the International Conference on Intelligent Robots and Systems (IROS'06), Beijing, China, October.

[7] S. Benhimane and E. Malis. A new approach to vision-based robot control with omni-directional cameras. In IEEE International Conference on Robotics and Automation (ICRA'06).

[8] Stefan Hrabar and Gaurav S. Sukhatme. A comparison of two camera configurations for optic-flow based navigation of a UAV through urban canyons. In Proceedings of the International Conference on Intelligent Robots and Systems (IROS'04).

[9] Stefan Hrabar and Gaurav S. Sukhatme. Omnidirectional vision for an autonomous helicopter. In in IEEE International Conference on Robotics and Automation (ICRA'03), pages 557-564.

[10] Geoff Cross Andrew W. Fitzgibbon and Andrew Zisserman. Automatic $3 \mathrm{~d}$ model construction for turn-table sequences. In Proceedings of SMILE Workshop on Structure from Multiple Images in Large Scale Environments, volume 1506 of Lecture Notes in Computer Science, pages 154-170, 1998.

[11] Reinhard Koch, Marc Pollefeys, and Luc J. Van Gool. Multi viewpoint stereo from uncalibrated video sequences. In Proceedings of the European Conference on Computer Vision (ECCV'98), pages 55-71.

[12] Branislav Mičušík, Daniel Martinec, and Tomáš Pajdla. 3d metric reconstruction from uncalibrated omnidirectional images. In Proc. of the Asian Conference on Computer Vision (ACCV '04).

[13] Petr Doubek and Tomáš Svoboda. Reliable 3d reconstruction from a few catadioptric images. In Proceedings of the Workshop on Omnidirectional Vision (Omnivis'02), pages 71-78.

[14] Maxime Lhuillier. Toward flexible 3d modeling using a catadioptric camera. In IEEE Conference on Computer Vision and Pattern Recognition (CVPR'07), pages 1-8.

[15] F. Dellaert, S. Seitz, C. Thorpe, and S. Thrun. Structure from motion without correspondence. In IEEE Conference on Computer Vision and Pattern Recognition (CVPR'O0), pages 557-564. 
[16] Ameesh Makadia and Kostas Daniilidis. Rotation recovery from spherical images without correspondences. IEEE Transactions on Pattern Analysis and Machine Intelligence (PAMI'06), 28(7):11701175.

[17] J. Kosecka and W. Zhang. Video compass. In Proceedings of European Conference on Computer Vision (ECCV'02), pages 657 - 673 .

[18] C. Rother. A new approach for vanishing point detection in architectural environments. In Proceedings of the British conference on Machine vision (BMVC'00), pages 382-391.

[19] Jean Charles Bazin, In So Kweon, Cedric Demonceaux, and Pascal Vasseur. Rectangle extraction in catadioptric images. In ICCV Workshop on Omnidirectional Vision, Camera Networks and Nonclassical Cameras (OMNIVIS'07).

[20] Matthew E. Antone and Seth J. Teller. Automatic recovery of relative camera rotations for urban scenes. In IEEE Computer Society Conference on Computer Vision and Pattern Recognition (CVPR'O0), pages 2282-2289.

[21] Patrick Rives and José R. Azinheira. Linear structures following by an airship using vanishing point and horizon line in a visual servoing scheme. In in IEEE International Conference on Robotics and Automation (ICRA'04), pages 255-260.

[22] Christopher Geyer and Kostas Daniilidis. Catadioptric projective geometry. International Journal of Computer Vision (IJCV'01), 45(3):223-243.

[23] M. J. Magee and J. K. Aggarwal. Determining vanishing points from perspective images. Computer Vision, Graphics and Image Processing, pages 256-267, 1984.

[24] L. Quan and R. Mohr. Determining perspective structures using hierarchical hough transform. Pattern Recognition Letters, 9(4):279286, 1989.

[25] Berthold K. P. Horn. Closed-form solution of absolute orientation using unit quaternions. Journal of the Optical Society of America. A, 4(4):629-642, Apr 1987.

[26] Jorge Lobo and Jorge Dias. Relative pose calibration between visual and inertial sensors. The International Journal of Robotics Research (IJRS'07), 26(6):561-575. 